\title{
Spatial-temporal mapping of hand, foot and mouth disease in relation to climate factors in Xinjiang, China from 2008 to 2016
}

ling xie ( $\sim 1428375964 @ q q . c o m)$

Xinjiang University https://orcid.org/0000-0002-1033-0420

Ruifang Huang

Xinjiang Uygur Autonomous region center for disease control and prevention

Hongwei Wang

Xinjiang University

Zhengqing Xiao

Beijing University of Posts and Telecommunications

Research article

Keywords: HFMD, air temperature, precipitation, spatial temporal analysis, Xinjiang Uygur Autonomous Region.

Posted Date: June 19th, 2020

DOI: https://doi.org/10.21203/rs.2.17027/v3

License: (c) (i) This work is licensed under a Creative Commons Attribution 4.0 International License. Read Full License 
1 Spatial-temporal mapping of hand, foot and mouth disease in relation to climate factors in Xinjiang, China from 2008 to 22016

$4 \quad{ }^{1}$ College of Resources and Environmental Sciences, Xinjiang University, Urumqi, 830046, China

$5 \quad{ }^{2}$ School of Environment and Resources, Guangxi Normal University, Guilin, 541004, China

$6 \quad{ }^{3}$ Xinjiang Uygur Autonomous Region Center for Disease Control and Prevention, Urumqi, 830001, China

$7 \quad{ }^{4}$ School of Computer Science \& Technology, Beijing University of Posts and Telecommunications, Beijing, 100875, China

9 [Objectives]: The study mainly aims to depict the epidemiological characteristics of hand, foot and mouth disease (HFMD) in

10 Xinjiang, China and to evaluate the effects of meteorological factors on the incidence of HFMD and the spatial-temporal

11 heterogeneity of HFMD in Xinjiang under the influence of meteorological factors.

12 [Methods]: With the data from the national surveillance data of HFMD and meteorological parameters in the study area from 2008

13 to 2016. We first employed GeoDetector Model to examine the effects of meteorological factors on HFMD incidence in Xinjiang,

14 China and to test the spatial-temporal heterogeneity of HFMD risk, and then the spatial autocorrelation was applied to examine the

15 temporal-spatial pattern of HFMD.

16 [Results]: From 2008 to 2016, the HFMD distribution showed a distinct seasonal pattern and HFMD cases typically occurred

17 between May and July, peaking in June, in Xinjiang. The relative humidity, precipitation, air pressure and temperature had more

18 influence than other risk factors on HFMD incidence with explanatory powers of $0.30,0.29,0.29$ and $0.21(\mathrm{p}<0.000)$, respectively.

19 The interactive effect of any two risk factors would enhance the risk of HFMD and there was a nonlinear enhancement between any

20 two risk factors interactive effect. The spatial relative risks in Northern Xinjiang were higher than in Southern Xinjiang. Global

21 spatial autocorrelation analysis showed a fluctuating trend over the years, the spatial dependency on the incidence of HFMD in 2008,

22 2010, 2012, 2014 and 2015, the negative spatial autocorrelation in 2009 and a random distribution pattern in 2011, 2013 and 2016.

23 [Conclusion]: Our findings show that the risk of HFMD in Xinjiang showed significant spatiotemporal heterogeneity. The monthly

24 average relative humidity, monthly average precipitation, monthly average air pressure and monthly average temperature factors

25 might have stronger relationships on the HFMD incidence in Xinjiang, China, compared with other factors. The differences in 
climate and latitude between Southern and Northern Xinjiang and their arid and semi-arid geographical environment are part of the 29 China.

reasons why the distribution of HFMD in Xinjiang is different from other temperate continental climatic zones. These associations draw attention to climate-related health issues and will help in establishing accurate spatiotemporal prevention of HFMD in Xinjiang,

Keywords: HFMD, meteorological factors, spatial-temporal analysis, GeoDetector, Xinjiang Uygur Autonomous Region.

\section{Background}

Hand, foot and mouth disease (HFMD) is an infectious disease related to various enteroviruses that mostly affect children below 5 years old [1]. Its pathogens are typically coxsackieviruses (coxsackievirus A16 (CVA16)) and enteroviruses (enterovirus 71 (EV71)) [2-5]. Its clinical manifestations mainly include mouth ulcers, fever, and vesicles on the hands, feet, and mouth[6]. Most HFMD patients can fully recover since HFMD is a self-limited disease, but some patients may develop severe life-threatening complications and even death[3]. HFMD is transmitted through direct contact with feces, respiratory droplets, and blister fluid of infective patients or the contaminated environment[5].

In recent years, HFMD outbreaks have been reported frequently in Asian countries such as Vietnam, Thailand, Singapore, Malaysia and China [7-8], causing widespread public health concerns. In 2014, China had an unprecedented number of large-scale outbreaks of HFMD, with the cumulative number of cases reaching 2,778,861[9]. In May 2008, HFMD was added to Category C of notifiable diseases for disease surveillance in China[10]. Thus, HFMD is increasingly widely concerned.

At present, several studies have extensively explored the correlation between HFMD and meteorological factors such as relative humidity [1,11-12], precipitation [12] and temperature [12-13]. The occurrence of HFMD showed apparent different characteristics in countries or regions with distinct climate conditions [14]. In Vietnam[15], HFMD increased 7\% (RR:1.07; 95\%CI: 1.052-1.088) and $3.1 \%$ (RR: $1.031,95 \% \mathrm{CI}: 1.024-1.039$ ) for $1{ }^{\circ} \mathrm{C}$ increase in monthly temperature above $26{ }^{\circ} \mathrm{C}$ and $1 \%$ increase in monthly humidity above $76 \%$. Whereas HFMD decreased $3.1 \%$ associated with a $1 \mathrm{~mm}$ increase in monthly cumulative rainfalls. In South Korea[16], at an average temperature below $18^{\circ} \mathrm{C}$, the HFMD rate increased by $10.3 \%$ for every $1{ }^{\circ} \mathrm{C}$ rise in average temperature ( $95 \%$ CI: $8.4,12.3 \%)$. We also saw a $6.6 \%$ increase in HFMD rate (95\% CI: 3.6, 9.7\%) with every $1 \%$ increase in relative humidity under 65\%, with a $1.5 \%$ decrease in HFMD rate observed (95\% CI: 0.4, 2.7\%) with each $1 \%$ humidity increase above $65 \%$. Annual 
51 in Sichuan, China [17]. The climatic types of arid and semi-arid regions in Northwest China are obviously different from the above

52 research areas. The basic quantitative study on meteorological factors and HFMD in Xinjiang is to explore the determinants of 53 meteorological driving factors of HFMD in Xinjiang.

To our knowledge, previous studies adopted different methods from different perspectives to explore the relationship between

HFMD incidence and meteorological factors. In summary, it can be divided into four categories: Establish a mathematical model

to predict the incidence of HFMD, for example, seasonal auto-regressive integrated moving average (SARIMA) models[3], SIR

model[18]. The risk model of HFMD was estimated by the variation of meteorological factors: time-series Poisson regression

models[5]. Meteorological factor changes and time lag model of HFMD: the generalized additive model [19], the negative binomial

multivariable regression model [20], the distributed lag non-linear model[21]. Determinants of the drivers of HFMD: spatial panel

data models [22], GeoDetector models [14]. To quantify the determinants powers of driving factors of HFMD in Xinjiang, the

\section{1}

62

Geographical detector is an appropriate model for this study. It is a set of statistical methods for detecting spatial differentiation and good at revealing the driving forces behind it.

Most of the previous studies concentrated in areas with high HFMD incidence, which are often characterized by high population density. The climate in high incidence areas is often temperate to humid, such as Hong Kong[20], Guangdong[13], Guangxi[23] and Jiangsu [24] provinces. However, the spatiotemporal heterogeneity of HFMD under the influence of meteorological factors in Northwest China was seldom studied. In general, the study on HFMD in arid and semi-arid regions is still limited; more research on such climates would potentially make the mechanism of HFMD transmission clearer under different climate conditions.

It was reported that the HFMD ranks the first in the number of class C infectious diseases in Xinjiang, 2019 [25], and the number of cases increases gradually each year, but the relationship between HFMD and meteorological factors in Xinjiang has not been proved. In this study, we analyze the spatial-temporal heterogeneity of HFMD and its relationship with meteorological factors in Xinjiang at the county scale, which is located in Northwest China having a semi-arid climate to shed more light on the mechanisms of HFMD transmission in those regions. The objectives of this study are: Firstly, to understand the spatiotemporal distribution characteristics of HFMD from 2008 to 2016 in Xinjiang, as well as the global spatial autocorrelation and the incidence of HFMD 
among different years. Secondly, factor detectors were used to quantify the determinants of meteorological drivers of HFMD, and risk detectors were used to detect the relative risks of HFMD under different meteorological elements. This study aims to provide countermeasures and suggestions for further public health interventions.

\section{Methods}

\subsection{Study area}

Xinjiang Uygur Autonomous Region is the largest provincial administrative region in China. The area is $166 * 10^{4} \mathrm{~km}^{2}$ and the population was 2486.76 million in 2018 . Xinjiang locates in the geographical center of Eurasia $\left(34.3^{\circ}-49.5^{\circ} \mathrm{N}, 73.5^{\circ}-96.3^{\circ} \mathrm{E}\right)$ and neighbors Russia, Kazakhstan, Kyrgyzstan, Tajikistan, Pakistan, Mongolia, India and Afghan from north to south. The mountains border Xinjiang on three sides and the Tianshan Mountains cuts across Northern Xinjiang. As a typical arid and semi-arid area, Xinjiang has a temperate continental climate. The annual mean temperature ranges from $9{ }^{\circ} \mathrm{C}$ to $12^{\circ} \mathrm{C}$ and the annual precipitations in Northern and Southern Xinjiang are respectively $210 \mathrm{~mm}$ and less than $100 \mathrm{~mm}$, displaying an uneven spatial distribution pattern.

The Tianshan Mountains have a higher level of precipitation, whereas Southern Xinjiang suffers severe water stress. The dominant wind throughout the year is northwest wind. Fig. 1 shows the geographical location of Xinjiang.

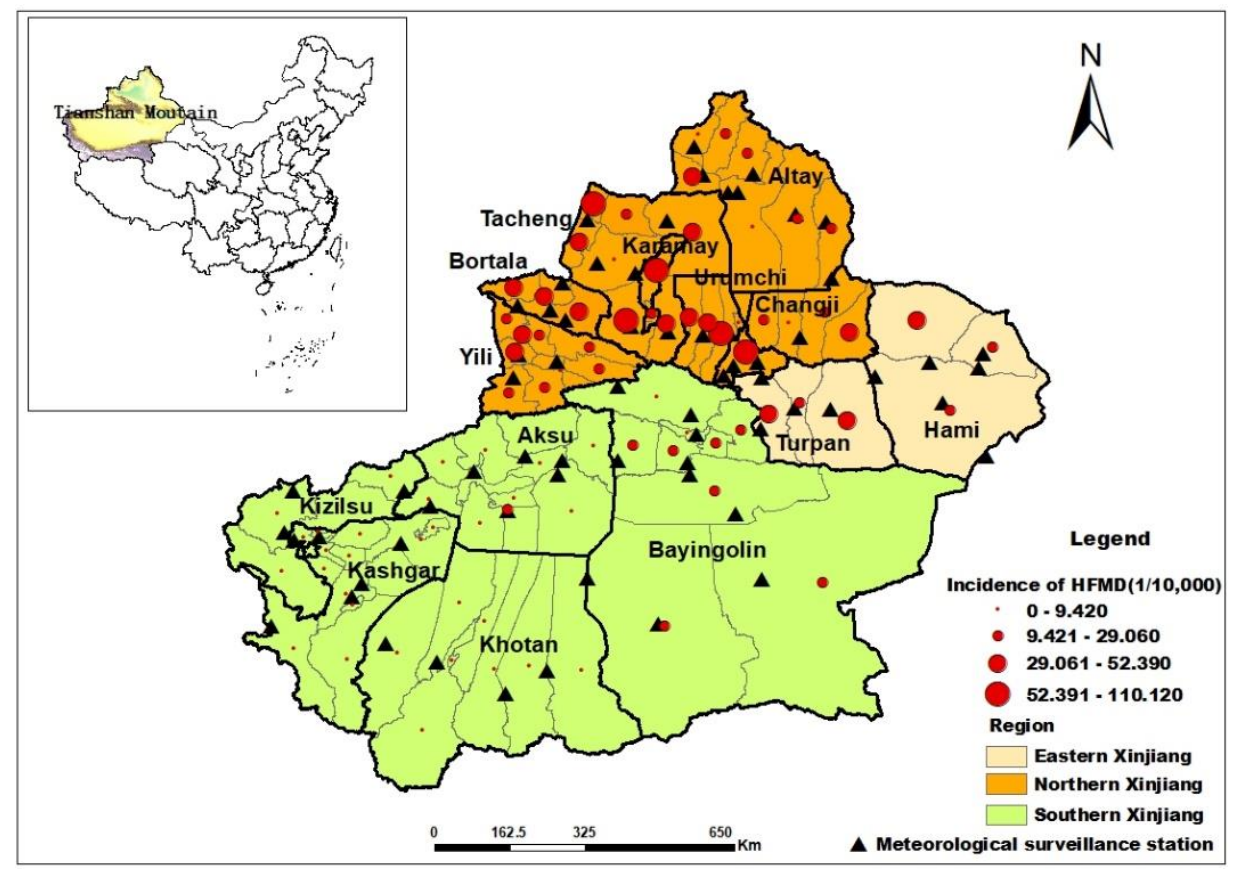

Fig. 1 Geographical location of the study area in China

\subsection{Data sources}


was reported to the CDC via the China Information System for Disease Control and Prevention, the national disease report system,

92 by the physicians treating the disease and public health personnel. The data of daily HFMD cases in Xinjiang from January 1,2008

93 to December 31, 2016 were from China Information System for Disease Control and Prevention. The collected patient's data include

94 gender, age, living address, types of patients, the onset date of symptom and confirmation time of symptom. Meteorological data were obtained from the China Meteorological Data Sharing Service System [25], including daily average temperature (TEM), daily average relative humidity (RHU), daily average barometric pressure (PRS), daily cumulative precipitation (PRE), daily average evaporation (EVP), daily average wind speed (WIN) and daily sunshine duration (SSD). Monthly average temperature, relative humidity, barometric pressure, precipitation, evaporation, wind speed and sunshine duration were computed or aggregated from daily weather data. The monthly county-level meteorological variables were estimated by using ordinary spatial kriging methods based on 66 meteorological surveillance stations within Xinjiang conducted by ArcGIS10.1. The 66 meteorological surveillance stations are mapped in Figure 1.

\subsection{Methods}

GeoDetector is a statistical method to detect the temporal-spatial heterogeneity, which quantitatively expresses the spatial stratified heterogeneity of the research object by analyzing the differences and similarities of intra-layer and inter-layer variance (http://www.geodetector.cn/). This tool has been widely used in land use, disease risk factor detection and other fields[27-28].

\section{Factor detector:}

In this study, the q-statistic in GeoDetector was used to quantify the spatiotemporal heterogeneity of HFMD and detect the interaction relationship between spatial effect and temporal effect [29]. GeoDetector was adopted to identify the risk factors from the 7 candidate meteorological factors that caused the temporal-spatial stratified heterogeneity of HFMD in Xinjiang from 2008 to 2016. The q-statistic was specified as follows Eq.1 (The q-statistic for heterogeneity analysis regarding covariates was provided by the R Geodetector software):

$$
q=1-\frac{1}{N \sigma^{2}} \sum_{h=1}^{L} N_{h} \sigma_{h}^{2}=1-\frac{S S W}{S S T} \quad S S W=\sum_{k=1}^{L} N_{k} \sigma_{k}^{2} S S T=N \sigma^{2}
$$


approaches 1 , it indicates a strong heterogeneity and if the value approaches 0 , it indicates a random distribution. $\mathrm{N}$ was the number

of all units, which could be divided into L strata. Stratum h was composed of $N_{h}$ units. SSW and SST are represented within the 116 sum of squares and the total sum of squares, $\sigma^{2}$ and $\sigma_{h}^{2}$ are the variance over all the units and within stratum $\mathrm{h}(\mathrm{h}=1, \ldots, \mathrm{L})$, 117 respectively.

\section{Interaction detector:}

By identifying the interactions between different risk factors $\mathrm{X}_{1}$ and $\mathrm{X}_{2}$, the interaction detector shows whether the interaction 121 each other. The relationship between the two factors can be divided into the following categories [30]:

$$
\text { Enhance: if } \mathrm{q}(\mathrm{X} 1 \cap \mathrm{X} 2)>\mathrm{q}(\mathrm{X} 1) \text { or } \mathrm{q}(\mathrm{X} 2)
$$

Enhance, bivariate: if $\mathrm{q}(\mathrm{X} 1 \cap \mathrm{X} 2)>\mathrm{q}(\mathrm{X} 1)$ and $\mathrm{q}(\mathrm{X} 2)$

Enhance, nonlinear: if $q(X 1 \cap X 2)>q(X 1)+q(X 2)$

Weaken: if $\mathrm{q}(\mathrm{X} 1 \cap \mathrm{X} 2)<\mathrm{q}(\mathrm{X} 1)+\mathrm{q}(\mathrm{X} 2)$

\section{Risk detector:}

area is divided into sub-regions according to the classification of each risk factor. The t statistics were used to determine whether there is a significant difference in the average incidence of HFMD between two sub-regions. The t statistic is shown in Eq 2:

$$
t_{y_{h=1}-\bar{y}_{h=2}}=\frac{\bar{Y}_{h=1}-\bar{Y}_{h=2}}{\left[\frac{\operatorname{Var}\left(\bar{Y}_{h=1}\right)}{n_{h=1}}+\frac{\operatorname{Var}\left(\bar{Y}_{h=2}\right)}{n_{h=2}}\right]^{1 / 2}}
$$

$\bar{Y}_{h}$ represents the mean value of attributes within sub-region h, such as incidence of HFMD; $n_{h}$ is the number of cases in 
Spatial autocorrelation analysis [31-32] including global spatial autocorrelation and local spatial autocorrelation, in this study,

we applied global spatial autocorrelation methods and the global Moran's I statistic to describe the spatial clusters of HFMD distribution in Xinjiang by GeoDa v1.2.0 software (http://geoda.uiuc.edu/downloadin.php). The Moran's I statistic is calculated by

Eq. (3)

$$
I=\left[n \sum_{i=1}^{n} \sum_{j=1}^{n} \omega_{i j}\left(X_{i}-\bar{X}\right)\left(X_{j}-\bar{X}\right)\right] /\left[\sum_{i=1}^{n} \sum_{j=1}^{n} \omega_{i j} \sum_{i=1}^{n}\left(X_{i}-\bar{X}\right)^{2}\right]
$$

where $n$ denotes the number of observed values, $X_{i}$ represents the incidence rate in the region $i, X_{j}$ represents the

143 incidence rate in the region $j, \bar{X}$ indicates the mean value, and $\omega_{i j}$ represents a spatial weight matrix of the systematic binomial distribution, which represents neighboring relations between geographical units with $\mathrm{n}$ representing the total number of those units. In the present study, the data were based on regions. The value $\omega_{i j}$ is 1 if region $i$ and region $j$ are adjacent.

\section{Otherwise, the value is 0 .}

The range of Moran's I value is $[-1,1]$ and the Moran scatter diagram represents the spatial agglomeration between a unit and

its surrounding units. When I $>0$, there is a positive spatial autocorrelation between space units within the range. In other words,

the difference in attribute values between adjacent space units is small. The distribution patterns of "low-low" clustering and "highand the smaller difference between attribute values is. When $\mathrm{I}<0$, there is negative spatial autocorrelation between spatial units within the range. In other words, there is a significant difference in the attribute values of adjacent spatial units and the closer I value is to -1 , the less concentrated distribution between spatial units is and the more significant difference in attribute values is. When I

$154=0$, there is no spatial autocorrelation between spatial units within the range and spatial variables present a random distribution 155 pattern.

\subsection{Descriptive analysis}



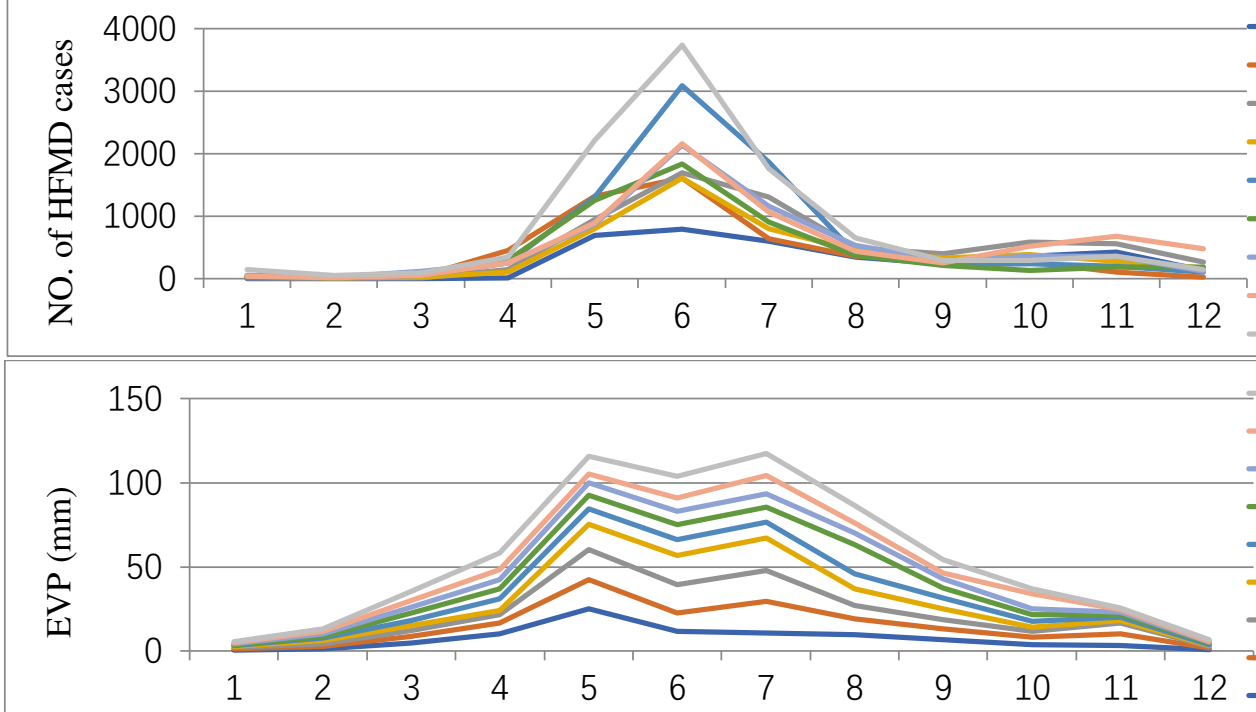

$-2011$

2012

$-2014$

2015

2016

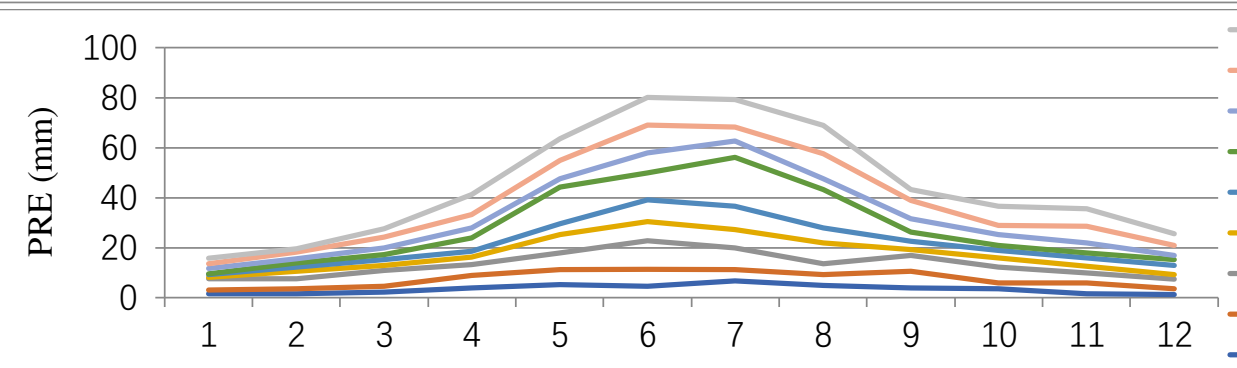

2016

$-2015$

$-2014$

-2013

-2012

2011

- 2010

$-2009$

2008
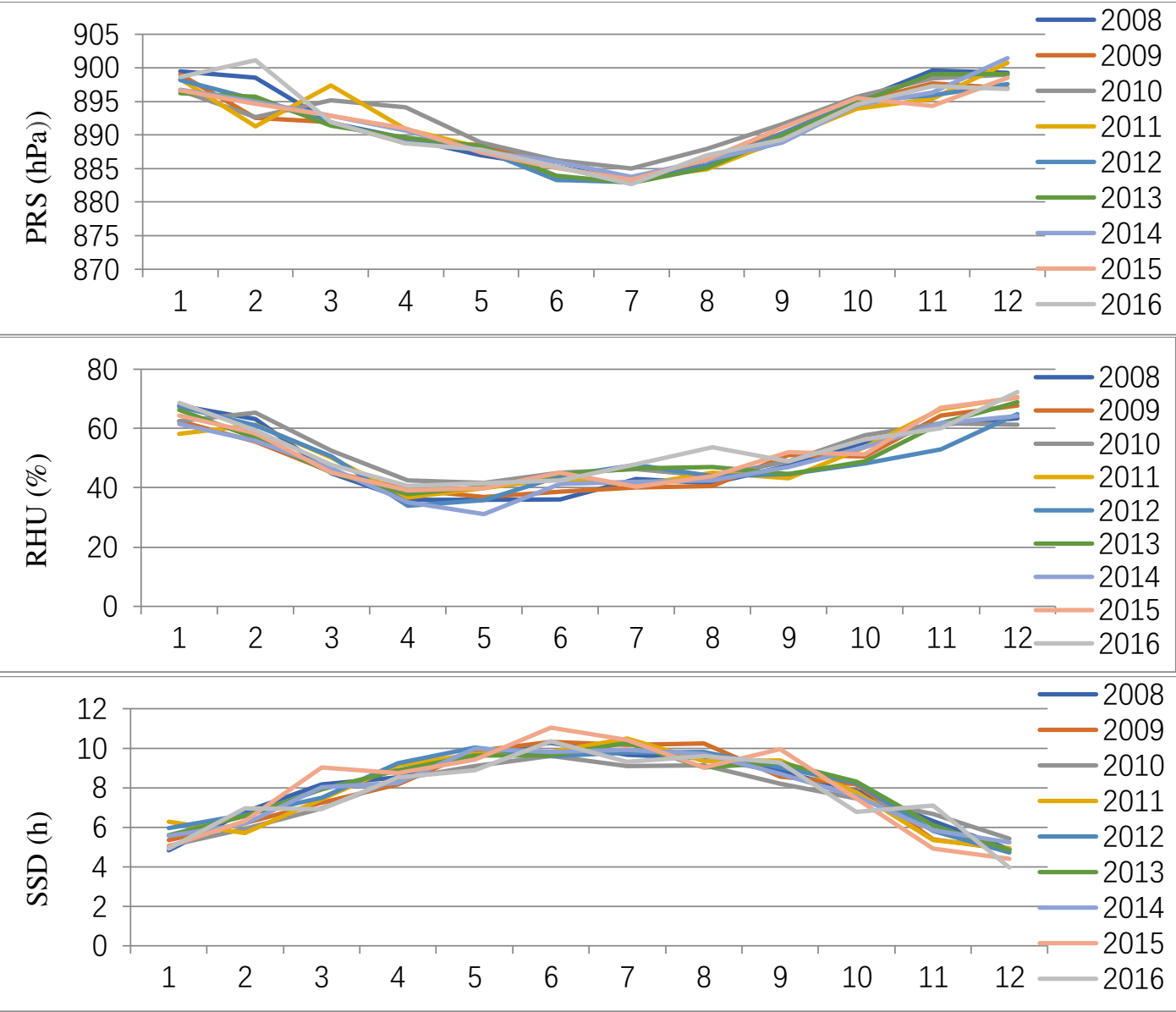


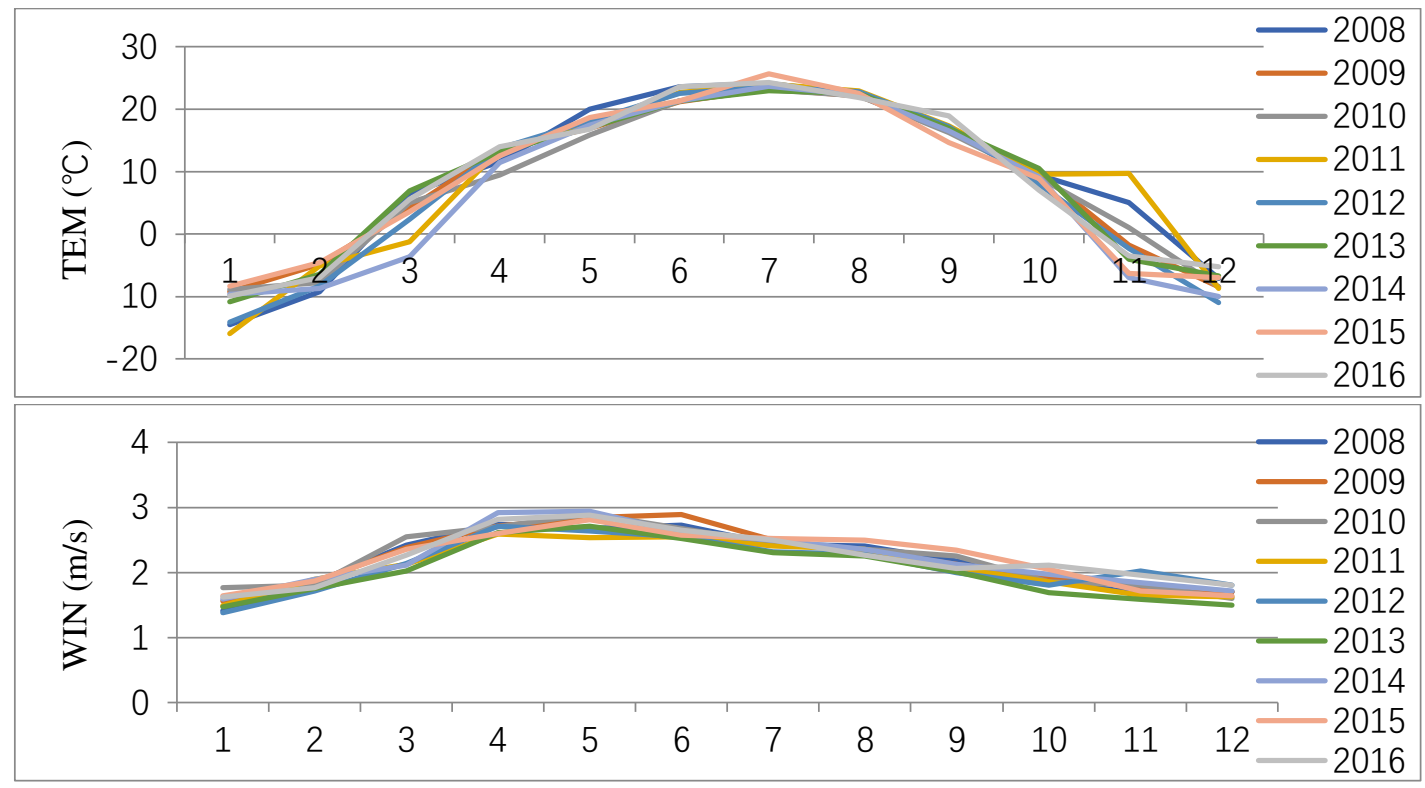

Fig. 2 Time series of monthly data of HFMD cases and meteorological variables in Xinjiang, China from 2008 to 2016

In total, 56,379 HFMD cases were reported from 2008 to 2016 in Xinjiang, with a daily average of 17.2 cases and the annual

average incidence was 25.27/10,000. Fig. 2 shows the monthly distributions of HFMD cases and meteorological variables in

Xinjiang from 2008 to 2016. The monthly HFMD distribution showed a distinct seasonal pattern over the period and HFMD cases

typically occurred between May and July, peaking in June. The annual morbidity among males was about 1.5 times higher than that

of females. Children under 5 years old were at the highest risk of HFMD. Most cases (86.2\%) were dispersed children who did not

go to kindergarten or school. A descriptive summary of the meteorological and socioeconomic variables is shown in Table 1.

Table 1 Descriptive statistics of meteorological variables

\begin{tabular}{lccccc}
\hline Covariates & Minimum & 25\% Percentile & Median & 75\% Percentile & Maximum \\
\hline Average relative humidity $(\%)$ & 32.35 & 41.64 & 51.52 & 61.11 & 70.69 \\
Average temperature $\left({ }^{\circ} \mathrm{C}\right)$ & 0.01 & 4.42 & 8.82 & 13.23 & 17.63 \\
Cumulative precipitation $(\mathrm{mm})$ & 0.02 & 0.49 & 0.95 & 1.42 & 1.91 \\
Sunshine duration (hour) & 5.72 & 6.8 & 7.89 & 8.97 & 10.05 \\
Average wind speed $(\mathrm{m} / \mathrm{s})$ & 0.84 & 1.68 & 2.53 & 3.37 & 4.21 \\
Average barometric pressure(hPa) & 699.42 & 777.75 & 856.08 & 934.41 & 1012.74 \\
Average evaporation $(\mathrm{mm})$ & 0.16 & 1.92 & 3.68 & 5.44 & 7.2 \\
\hline
\end{tabular}

\subsubsection{Factor detector analysis}

As shown in Table 2, the determinant power of the average relative humidity was obviously associated with the incidence of

$\operatorname{HFMD}(q=0.30)$, indicating that the average relative humidity mainly explains the spatial heterogeneity of the incidence of HFMD.

Precipitation, barometric pressure, temperature and sunshine duration were also associated with the incidence of HFMD in Xinjiang, 
Table 2 Explanatory power of each impact factor on the incidence of HFMD in Xinjiang

\begin{tabular}{lllllllll}
\hline & EVP & PRE & PRS & RHU & SSD & TEM & WIN \\
\hline q statistic & 0.11 & 0.29 & 0.29 & 0.30 & 0.20 & 0.21 & 0.07 \\
p value & 0.000 & 0.000 & 0.000 & 0.000 & 0.005 & 0.000 & 0.005 \\
\hline
\end{tabular}

\section{1}

\subsubsection{Interaction detector:}

The study found that the interaction of any two risk factors has greater explanatory power than any single metrological factor.

Compared with their individual impact, they most presented the effect of "nonlinear enhance" or "bivariate enhance". As shown in

Table 3, the q statistics of average relative humidity was 0.3 , which increased to 0.5 after accounting for the interactive effect of average barometric pressure on the HFMD incidence. As 0.5 is significantly higher than 0.3 (q statistics of average relative humidity) and 0.29 (q statistics of average barometric pressure), the result indicated that relative humidity and barometric pressure has a significantly bivariate enhanced interactive associations on the incidence rate of HFMD. The explanatory power of average relative humidity increased to 0.39 after considering the interactive effect of precipitation on HFMD incidence. The coupled impact of average relative humidity $(\mathrm{q}=0.3)$ and average wind speed $(\mathrm{q}=0.07)$ played an important role in HFMD, with an explanatory power of 0.43 (Table 3). High average relative humidity and high average wind speed were associated with a high incidence of HFMD. The interaction of these risk factors could effectively explain the spatial heterogeneity of the HFMD, and the selected risk factors had a tendency of strengthening interaction.

Table 3 The value of $\mathrm{q}$ for interactions between pairs of factors on the incidence of HFMD.

\begin{tabular}{cccccccc}
\hline Covariates & EVP & PRE & PRS & RHU & SSD & TEM & WIN \\
\hline EVP & 0.11 & & & & & & \\
PRE & $0.34 \mathrm{~EB}$ & 0.29 & & & & & \\
PRS & $0.35 \mathrm{~EB}$ & $0.37 \mathrm{~EB}$ & 0.29 & & & & \\
RHU & $0.35 \mathrm{~EB}$ & $0.39 \mathrm{~EB}$ & $0.50 \mathrm{~EB}$ & 0.30 & & & \\
SSD & $0.21 \mathrm{E}$ & $0.33 \mathrm{~EB}$ & $0.40 \mathrm{~EB}$ & $0.38 \mathrm{~EB}$ & 0.20 & & \\
TEM & $0.34 \mathrm{EN}$ & $0.33 \mathrm{~EB}$ & $0.41 \mathrm{~EB}$ & $0.37 \mathrm{~EB}$ & $0.34 \mathrm{~EB}$ & 0.21 & \\
WIN & $0.25 \mathrm{EN}$ & $0.36 \mathrm{ID}$ & $0.36 \mathrm{ID}$ & $0.43 \mathrm{EN}$ & $0.35 \mathrm{EN}$ & $0.32 \mathrm{EN}$ & 0.07 \\
\hline
\end{tabular}

Note: EN: Enhance (nonlinear), EB: Enhance (bivariate), ID: Independent, E: Enhance

\subsubsection{Risk detector analysis}

Figure 3 shows the spatial distribution of HFMD cases in Xinjiang from 2008 to 2016, the number of HFMD cases was divided into four grades by natural breaks using ArcGIS. A deeper red color indicates a higher number of HFMD cases, most of them were concentrated in Northern Xinjiang. 
the distribution of RR in space was not the same every year, and there were certain changes. Specifically, the spatial RRs in counties

in Northern Xinjiang were higher than the counties in Southern Xinjiang, implying that these counties have relatively higher HFMD risk. Conversely, counties in Southern Xinjiang generally have lower RRs. This is consistent with the spatial distribution characteristics of the number of HFMD cases in Figure 3. The Northern Xinjiang had a higher average relative humidity, suitable temperature and precipitation level, resulting in a higher RR of HFMD. The southern regions were affected by the Taklimakan desert, high temperature, low relative humidity, precipitation and air pressure, and the risk of HFMD is relatively low. We found that the lowest RR of HFMD in Khotan, during the study period. According to the following meteorological risk factor charts,
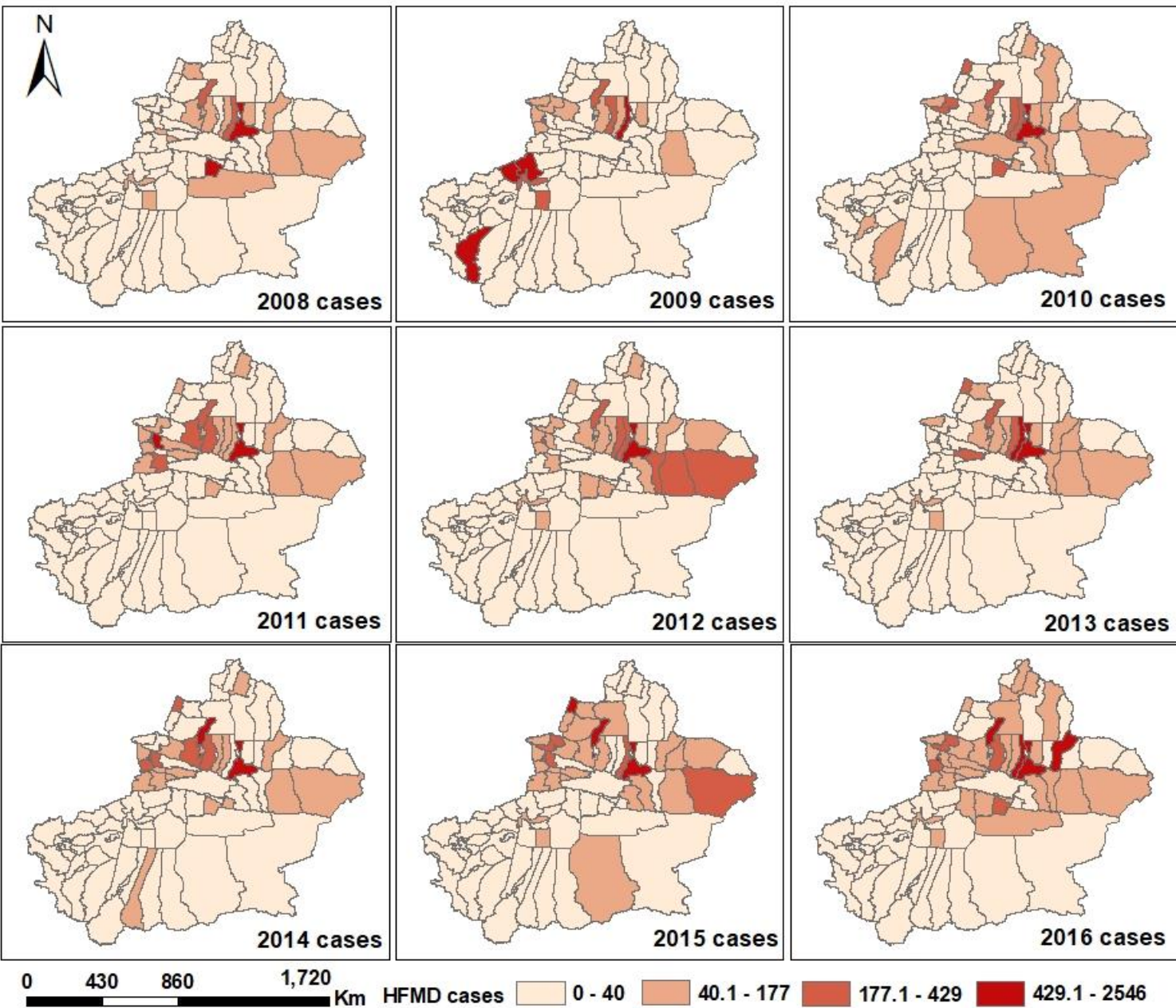

HFMD cases

$0-40$

40.1 - 177

177.1 - 429

429.1 - 2546

Fig. 3 The spatial distribution of HFMD cases in Xinjiang 

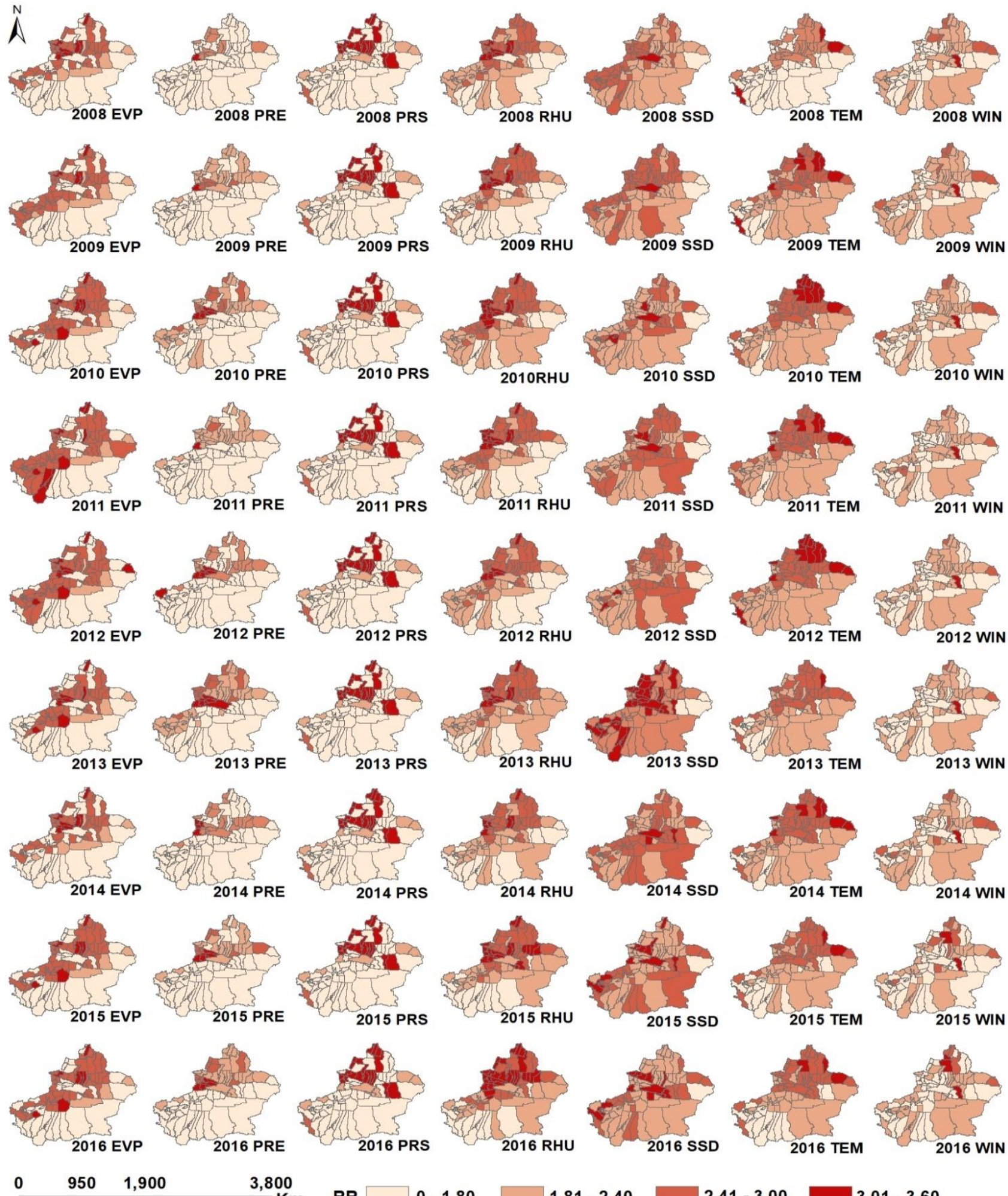

$R R \square 0-1.80$

$1.81-2.40$

$2.41-3.00$

$3.01-3.60$

Fig. 4 The relative risk (RR) values of the spatiotemporal interaction effect

In Figure 5 we found that when the monthly average precipitation exceeded $0.94 \mathrm{~mm}$, the incidence of HFMD decreased. The 
and HFMD. When the monthly average relative humidity ranges from $51.52 \%$ to $70.69 \%$, HFMD shows a higher incidence than other periods. 6.8 to 8.96 hours of sunshine duration are the sensitive monthly average sunshine duration for HFMD. When the monthly average temperature was $8.81^{\circ} \mathrm{C}$, the HFMD reached a peak. The incidence of HFMD was the most active during the temperature changed from $4.22^{\circ} \mathrm{C}$ to $13.22^{\circ} \mathrm{C}$. The incidence of HFMD increased along with the monthly average relative humidity and the monthly average sunshine duration, reached a peak when the monthly average relative humidity was at $61.1 \%$ and the monthly average sunshine duration was at 7.78 hours and then decreased afterwards, respectively. Risk detector value presented a logarithmic relationship between the monthly average evaporation and HFMD, an exponential relationship between the monthly mean air pressure and HFMD. With regards to the association between the monthly average wind speed and HFMD, the incidence of HFMD was the highest when the monthly average wind speed was less than $2.52 \mathrm{~m} / \mathrm{s}$. It should be noted that when a single meteorological factor mentioned above reaches the high incidence period of HFMD, it does not mean the outbreak period of HFMD,

and it also requires the comprehensive action of other meteorological factors.
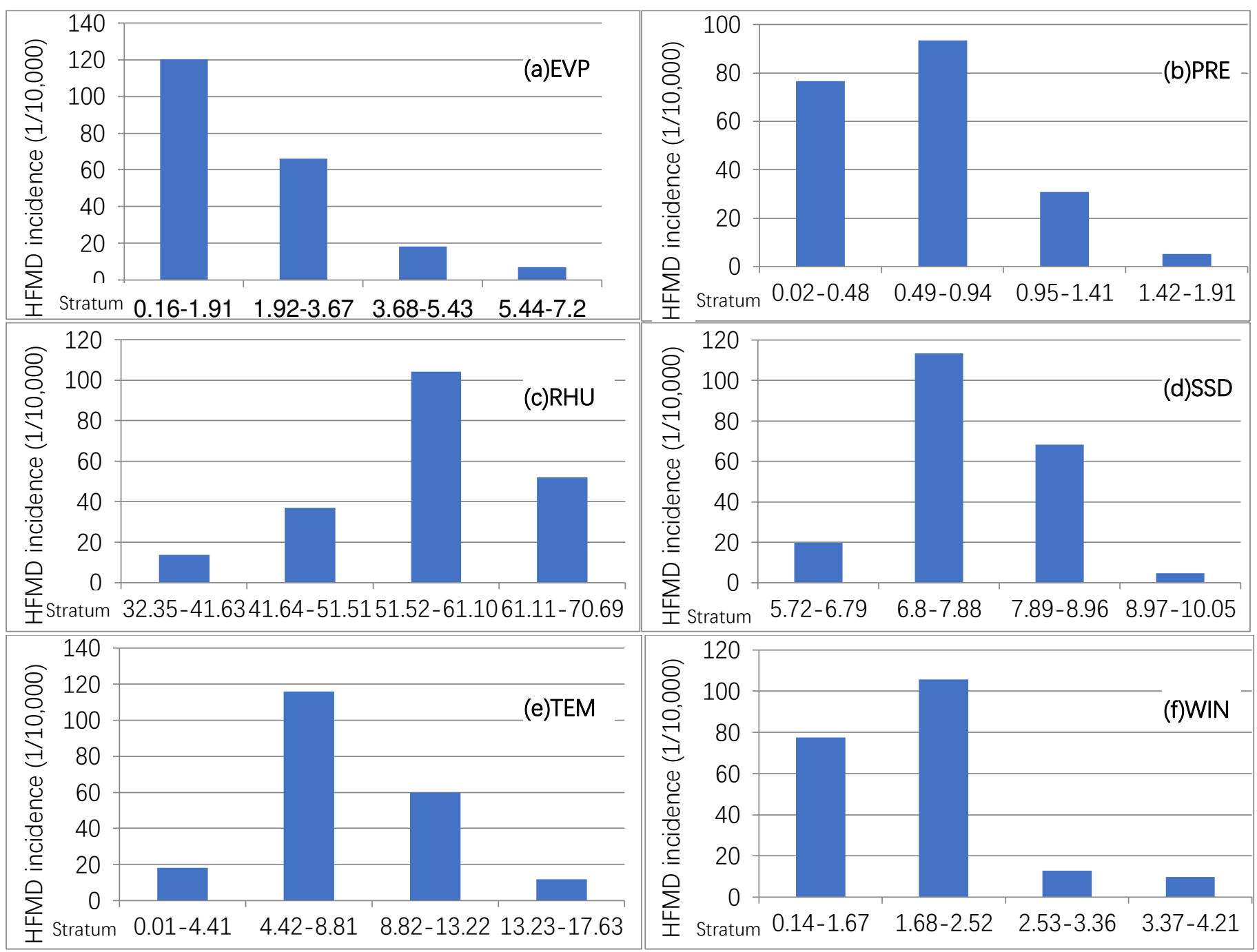


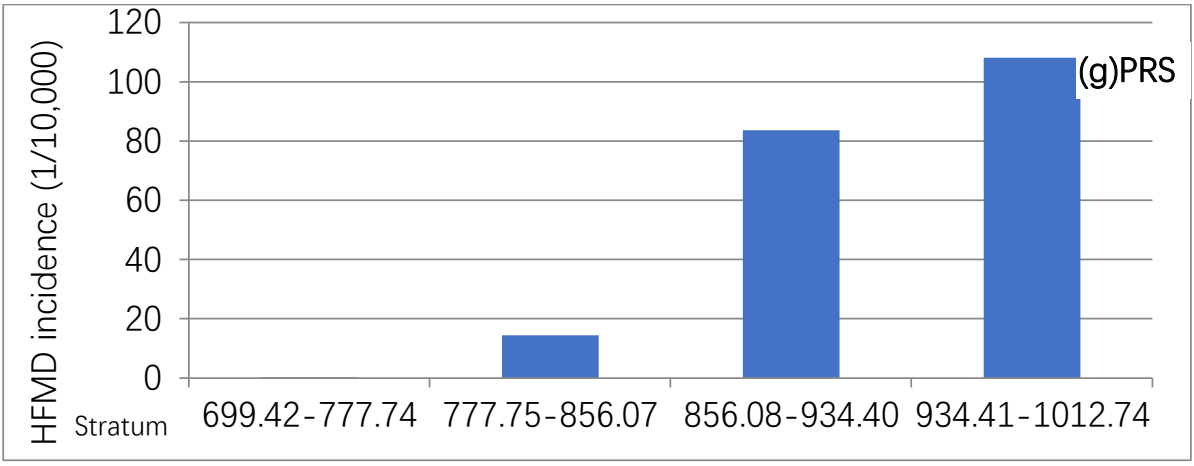

Fig. 5 Average incidence of HFMD according to the different risk factor strata. a. Evaporation; b. Precipitation; c. Relative

\subsection{Spatial autocorrelation of HFMD incidence}

Moran's I value was calculated by global spatial autocorrelation analysis. From 2008 to 2016, the Moran's I value (Table 4) of

HFMD in Xinjiang ranged from -0.135 to $0.202(\mathrm{P}<0.05)$, showing a fluctuating trend over the years, indicating the spatial dependency on the occurrence of HFMD in 2008, 2010, 2012, 2014 and 2015. Moran's I value in 2009 was -0.135, indicating that there was a negative spatial autocorrelation of HFMD in Xinjiang. Moran's I values in 2011 and 2016 were respectively -0.066 and

-0.00018 , indicating that the incidence of HFMD in Xinjiang presented a random distribution pattern. Bayingolin Mongol

Autonomous Prefecture showed the high-high spatial autocorrelation of HFMD incidence, whereas Kashgar, Hotan, Aksu and

Kizilsu Kirghiz Autonomous Prefecture showed the low-low spatial autocorrelation of HFMD incidence in 2008 and 2010. From

2011 to 2016, Urumqi always showed the high-high spatial autocorrelation of HFMD incidence.

Table 4 Results of the spatial autocorrelation test on HFMD cases in Xinjiang from 2008 to 2016.

\begin{tabular}{llllllllll}
\hline Years & 2008 & 2009 & 2010 & 2011 & 2012 & 2013 & 2014 & 2015 & 2016 \\
\hline Moran's I & 0.144 & -0.135 & 0.202 & -0.066 & 0.120 & 0.056 & 0.134 & 0.146 & -0.001 \\
Z-score & 1.904 & -0.647 & 2.832 & 0.042 & 1.933 & 1.181 & 2.092 & 2.146 & 0.625 \\
P values & $P<0.05$ & $P<0.05$ & $P<0.05$ & $P<0.05$ & $P<0.05$ & $P<0.05$ & $P<0.05$ & $P<0.05$ & $P<0.05$ \\
\hline
\end{tabular}

different regions, the HFMD risk has introduced a significant difference. The correlation between HFMD incidence and climatic

factors has been extensively explored[6,15], the relationships between HFMD occurrence and various meteorological factors in

Xinjiang where typical arid and semi-arid areas were interpreted with daily HFMD surveillance data for the first time in the study. 
conditions.

Previous studies have shown that the response of HFMD incidence with respect to climate change differs. In temperate regions,

253 HFMD outbreaks usually occurred in summer or early fall [5] since temperature rise and precipitation increase occurred later than in tropical regions. Therefore, HFMD incidence in temperate regions was different from that in tropical and subtropical regions

[19,34]. For example, a study conducted in Vietnam located in Southeast Asia showed that the peak of HFMD comes in April.

However, a study of HFMD in Gansu, China, showed that the HFMD reached the peak in June [35]. The climate of the two places

mentioned above differs markedly. The study on the response of meteorological factors to HFMD has some similarities and

differences with the previous conclusions. For instance, a study conducted in Guangdong province located in South China, showed

that there was a nonlinear relationship between temperature and HFMD [13]. The positive correlation between temperature increase

and HFMD incidence are corroborated by other studies. For instance, a study in Japan, with the increase in temperature, Herpangina

\& HFMD incidence increased. In a warm environment, the transmission of the HFMD virus was enhanced, but cold and hot climate

limited the transmission [5,6,24].Similarly, a study was implemented in Ningxia which located in the upper reaches of the Yellow

River in Western China, it demonstrated that average temperature, relative humidity, and wind speed played significant roles in the

spatial-temporal distribution of HFMD risk [14]. In our research, the relationship between monthly average temperature and HFMD

relative risk represents inverted $\mathrm{V}$-shape, a similar pattern was observed for the association between the monthly average relative

humidity and HFMD, the monthly average precipitation and HFMD, the monthly average sunshine duration and HFMD, the monthly

average wind speed and HFMD. It indicates that there is a non-linear relationship between meteorological factors and the risk of

HFMD in Xinjiang. Furthermore, another study in Guangdong which has a high incidence of HFMD in China, indicated that the

risk of HFMD was significantly associated with monthly average relative humidity, monthly average temperature, and monthly

average rainfall. In this study, from the results of GeoDetector, an increase in HFMD incidence was found to be associated with

the monthly average relative humidity, monthly average precipitation, monthly average air pressure and monthly average

temperature, which was similar to some other research studies. Other climate conditions may also have a threshold effect. It should 
to a higher risk of HFMD [37]. However, the monthly average relative humidity between 51.52 to $61.10 \%$, which is the threshold

of HFMD in Xinjiang. Ultimately, we analyzed the association between temperature, precipitation and the incidence of HFMD in

Xinjiang Uygur Autonomous Region, China, from one previous study, which can be used to support our results [38]. It's worth noting that risk detector value presented a logarithmic relationship between the monthly average evaporation and HFMD, an

exponential relationship between the monthly mean air pressure and HFMD. This is different from previous research results.

The possible reason of the relationship between HFMD and meteorological factors is the stability of the enterovirus that causes

HFMD in the external environment, such as humidity and temperature [39]. Moderate monthly cumulative precipitation can sustain

the prevalence of HFMD. Because precipitation might affect water sanitation, promote the attachment of HFMD virus and increase

the risk of exposure, thus facilitating the spread of HFMD and previous studies also have strong support for this view $[1,12]$.

In order to further explore the relationship between meteorological factors and HFMD incidence, in this study, GeoDetector was adopted to explore the spatial and temporal heterogeneity and its interactive effects on the HFMD incidence in Xinjiang and spatial autocorrelation analysis was applied to examine the spatial pattern of HFMD in Xinjiang. The results demonstrated that

HFMD incidence showed obvious regional differentiation, displaying a dynamic spatial-temporal distribution. The distribution of

HFMD was mainly concentrated in Northern Xinjiang. The incidence of HFMD in Southern Xinjiang might be ascribed to the

precipitation stress. The incidence of HFMD in Urumqi, Changji Prefecture, Tacheng Prefecture and other areas in Northern

Xinjiang was obviously worthy of in-depth investigation. We think the possible reason is these focal areas have a well-developed economy, the fast highway system, and the large heterogeneous migrant population, which increases the HFMD risk [17].

The global spatial autocorrelation analysis results demonstrated that the area with the high HFMD incidence was different from the high spatial autocorrelation area of HFMD in Xinjiang. Urumqi has always shown the high spatial autocorrelation of HFMD incidence because the incidence of HFMD in Urumqi remained high over the years and the adjacent area also had a high incidence of HFMD. Multiple regions showed low spatial autocorrelation because the incidence of HFMD had inter-annual variation in several regions, with a low incidence of HFMD in adjacent areas, such as Aksu and Ili Kazak Autonomous Prefecture. 
more accurate than using the same meteorological data on all counties in each city. Second, we estimated spatiotemporal variations

in HFMD at the scale of counties and months; we did not include the factors at an individual and pathogenic levels, such as personal

hygiene, educational background, incomes of the children's parents, living conditions, and composition of major pathogens. Further

studies should consider the potential impacts of these factors. Third, the result should be examined via different investigation

techniques, such as geographically weighted regression (GWR). With GWR, spatially changing relationships between variables can

be explored [41,42]. GWR will be applied in our study on the relationship between HFMD incidence and potential impact factors.

\section{Conclusions}

The findings of our study indicated that the spatial-temporal distribution of HFMD risk in Xinjiang, China, was non-

homogeneous. The higher relative risk counties mainly gathered in Northern Xinjiang. Meteorological factors, such as monthly

average relative humidity, monthly average precipitation, monthly average barometric pressure and monthly average temperature,

might be the driving factors of HFMD in Xinjiang. The high relative risk areas and high spatial correlation areas of HFMD all point

to the Northern Xinjiang. Therefore, in the high incidence of HFMD season and relative risk areas, we can reduce environmental

exposure and contact transmission to decrease the spread of HFMD.

\section{Abbreviations}

HFMD: Hand, foot and mouth disease; CA16: coxsackievirus A16; EV71: enterovirus 71; RR: relative risk; CDC: Chinese Centre for Disease Control and Prevention; TEM: temperature; RHU: relative humidity; PRS: pressure, PRE: precipitation; EVP: evaporation; WIN: wind speed; SSD: sunshine duration; EN: Enhance (nonlinear); EB: Enhance (bivariate); ID: Independent; E: Enhance.

\section{Acknowledgements}

We would like to extend our thanks to Xinjiang Center for Disease Control and Prevention, China for sharing data on Xinjiang hand, foot and mouth disease not referring to patient's privacy information.

\section{Authors' Contributions}

HW W were in charge of the conception, design of study and revised the manuscript. L X wrote the manuscript and revised it critically for intellectual content. ZQ X undertook in data analyzed and helped to revise the manuscript. RF H provided HFMD data. All authors read and approved the final manuscript.

\section{Availability of data and materials}

The meteorological data used in the study were available from National Meteorological Information Center (http://data.cma.cn/). The vector map data was applied from Resource and Environment Data Cloud(http://www.resdc.cn/) with official permission. The datasets of HFMD generated during and analyzed during the current study are not publicly available due to confidentiality requirements but are available from the corresponding author on reasonable request. 


\section{Competing interests}

The authors declare that they have no competing interests.

\section{Consent to publish}

Not applicable.

\section{Ethics approval and consent to participate}

This study was reviewed and approved by the Ethics Committee of the Xinjiang Center for Disease Control and Prevention, China. The authors assert that all procedures contributing to this work comply with the ethical standards of the relevant national and institutional committees. We aggregated the HFMD for each day at the county level (excluding the Xinjiang production and construction corps), not referring to patient's privacy information.

\section{Funding}

This work was supported by the National Natural Science Fund of China (Grant No 4186010245), Additionally, the funding body in the study had no role in the design of the study and collection, analysis, and interpretation of data and in writing the manuscript.

\section{Reference}

1. Chen C, Lin H, Li X, Lang L, et al. Short-term effects of meteorological factors on children hand, foot and mouth disease in Guangzhou, China. Int J Biometeorol. 2014, 58:1605-1614.

2. Chen S, Yang D, Liu R, et al. Estimating the transmissibility of hand, foot, and mouth disease by a dynamic model. Public Health. 2019, 174:42-48.

3. Ma E, Lam T, Wong C, Chuang SK. Is hand, foot and mouth disease associated with meteorological parameters? Epidemiol Infect. 2010, 138:1779-1788.

4. Nguyen HX, Chu C, Nguyen HLT, et al. Temporal and spatial analysis of hand, foot, and mouth disease in relation to climate factors: A study in the Mekong delta region, Vietnam. The Science of the total environment. 2017, 581-582:766772.

5. Yien Ling Hii, Joacim Rocklov, Ng N. 2011. Short term effects of weather on hand, foot and mouth disease. PLoS One. 2011, 6(2):e16796

6. Onozuka D, Hashizume M. The influence of temperature and humidity on the incidence of hand, foot, and mouth disease in japan. The Science of the total environment. 2011, 410-411:119-125.

7. Wang $Y$, Feng $Z$, Yang $Y$, et al. Hand, foot, and mouth disease in china: Patterns of spread and transmissibility. Epidemiology. 2011, 22:781-792.

8. Xing, W., Q. Liao, C. Viboud, J. et al. Hand, foot, and mouth disease in China, 2008-12: an epidemiological study. The Lancet Infectious Diseases. 2014, 14(4): 308-318.

9. http://www.phsciencedata.cn/

10. Qunying Mao, Yiping Wang, Liang Z. Hand, foot, and mouth disease in mainland china. The lancet infection disease. 2014, 14:1041.

11. Xu C, Zhang X, Xiao G. Spatiotemporal decomposition and risk determinants of hand, foot and mouth disease in Henan, China. The Science of the total environment. 2019, 657:509-516.

12. Nguyen, H. X., C. Chu, Q. D. Tran, et al. Temporal relationships between climate variables and hand-foot-mouth disease: a multi-province study in the Mekong Delta Region, Vietnam. International Journal of Biometeorology. 2019,12 e:018249

13. Xu, Z., W. Hu, K. Jiao, et al. The effect of temperature on childhood hand, foot and mouth disease in Guangdong Province, China, 2010-2013: a multicity study. BMC Infectious Diseases. 2019, 19(1):969

14. Li, J., X. Zhang, L. Wang, et al. Spatial-temporal heterogeneity of hand, foot and mouth disease and impact of meteorological factors in arid/ semi-arid regions: a case study in Ningxia, China. BMC Public Health. 2019, 19(1):1482

15. Phung D, Nguyen HX, Nguyen HLT, et al. Spatiotemporal variation of hand-foot-mouth disease in relation to socioecological factors: A multiple-province analysis in Vietnam. The Science of the total environment. 2018, 610- 
611:983-991.

16. Munderloh UG, Kim Bl, Ki H, et al. Effect of climatic factors on hand, foot, and mouth disease in South Korea, 2010 2013. Plos One. 2016, 11: e0157500.

17. Liao J, Qin Z, Zuo Z, et al. Spatial-temporal mapping of hand foot and mouth disease and the long-term effects associated with climate and socio-economic variables in Sichuan province, china from 2009 to 2013. The Science of the total environment. 2016, 563-564:152-159.

18. Du, Z., W. Zhang, D. Zhang, et al. Estimating the basic reproduction rate of HFMD using the time series SIR model in Guangdong, China. PLoS One. 2017, 12(7): e0179623.

19. Xu J, Zhao D, Su H, et al. Impact of temperature variability on childhood hand, foot and mouth disease in Huainan, china. Public Health 2016, 134:86-94.

20. Wang P, Goggins WB, Chan EY. 2016. Hand, foot and mouth disease in Hong kong: A time-series analysis on its relationship with weather. PLoS One. 11: e0161006.

21. Zhang Z, Xie X, Chen X, et al. Short-term effects of meteorological factors on hand, foot and mouth disease among children in Shenzhen, China: Non-linearity, threshold and interaction. The Science of the total environment. 2016, 539:576-582.

22. Wang H, Du Z, Wang $X$, et al. Detecting the association between meteorological factors and hand, foot, and mouth disease using spatial panel data models. Int J Infect Dis. 2015, 34:66-70.

23. Liu, H., G. Song, N. He, et al. Spatial-temporal variation and risk factor analysis of hand, foot, and mouth disease in children under 5 years old in Guangxi, China. BMC Public Health. 2019, 19(1): 1491.

24. Liu W, Ji H, Shan J, et al. Spatiotemporal dynamics of hand-foot mouth disease and its relationship with meteorological factors in Jiangsu province, China. PLoS One. 2015,6(29):e 0131311

25. http://www.xjhfpc.gov.cn/

26. http://data.cma.cn/

27. http://www.geodetector.cn/

28. Wang, J.-F. et al. Geographical Detectors-Based Health Risk Assessment and its Application in the Neural Tube Defects Study of the Heshun Region, China. International Journal of Geographical Information Science ,2010,24, 107-127

29. Gu, J., L. Liang, H. Song, et al. A method for hand-foot-mouth disease prediction using GeoDetector and LSTM model in Guangxi, China. Sci Rep. 2019, 9(1): 17928.

30. Wang, J. Zhang, T. Fu, B. A measure of spatial striated heterogeneity. Ecol. Indic. 2016, 67, 250-256.

31. WANG J F, XU C D. Geodetector: Principle and prospective []]. Acta Geographica Sinica, 2017, 72(1): 116-134

32. Mao, Y., N. Zhang, B. Zhu, et al. A descriptive analysis of the Spatio-temporal distribution of intestinal infectious diseases in China." BMC Infect Dis. 2019, 19(1): 766.

33. H Y Zhang, L. Y., L P Li, et al. The epidemic characteristics and spatial autocorrelation analysis of hand, foot and mouth disease from 2010 to 2015 in Shantou, Guangdong, China. 2019

34. Huang, R., H. Ning, T. He, et al. Impact of PM10 and meteorological factors on the incidence of hand, foot, and mouth disease in female children in Ningbo, China: a spatiotemporal and time-series study. Environmental Science and Pollution Research. 2017, 26(18): 17974-17985.

35. Gou, F., X. Liu, J. He, et al. Different responses of weather factors on hand, foot and mouth disease in three different climate areas of Gansu, China. BMC Infect Dis .2018,18(1): 15.

36. Wang, Y., Y. Lai, Z. Du, et al. Spatiotemporal Distribution of Hand, Foot, and Mouth Disease in Guangdong Province, China and Potential Predictors, 2009-2012. Int J Environ Res Public Health. 2019,16(7):1191.

37. Du Z C, Zhang W J, Zhang DM, et al. The threshold effects of meteorological factors on hand, foot, and mouth disease (HFMD) in China, 2011. Sci Rep. 2016, 6:36351.

38. Huang RF, Xie L, Liu SH, et al. Correlation analysis on meteorological factors regarding the incidence of hand, foot and mouth disease in Xinjiang Uygur Autonomous Region, 2011-2018. Chinese Journal of Epidemiology, 2019, 40(12): 1563-1568

39. Wei J, Hansen A, Liu Q, et al. The effect of meteorological variables on the transmission of hand, foot and mouth disease in four major cities of Shanxi province, china: A time series data analysis (2009-2013). PLoS Negl Trop Dis. 
2015, 9: e0003572.

40. Zhu L, Yuan Z, Wang X, et al. The impact of ambient temperature on childhood HFMD incidence in inland and coastal area: a Two-City study in Shandong province, China. Int J Environ Res Public Health. 2015, 12(8):8691-704.

41. Gilbert A, Chakraborty J. Using geographically weighted regression for environmental justice analysis: Cumulative cancer risks from air toxics in florida. Social Science Research 2011, 40:273-286.

42. Rybnikova N, Stevens RG, Gregorio DI, et al. Kernel density analysis reveals a halo pattern of breast cancer incidence in connecticut. Spat Spatiotemporal Epidemiol. 2018, 26:143-151. 
Figures

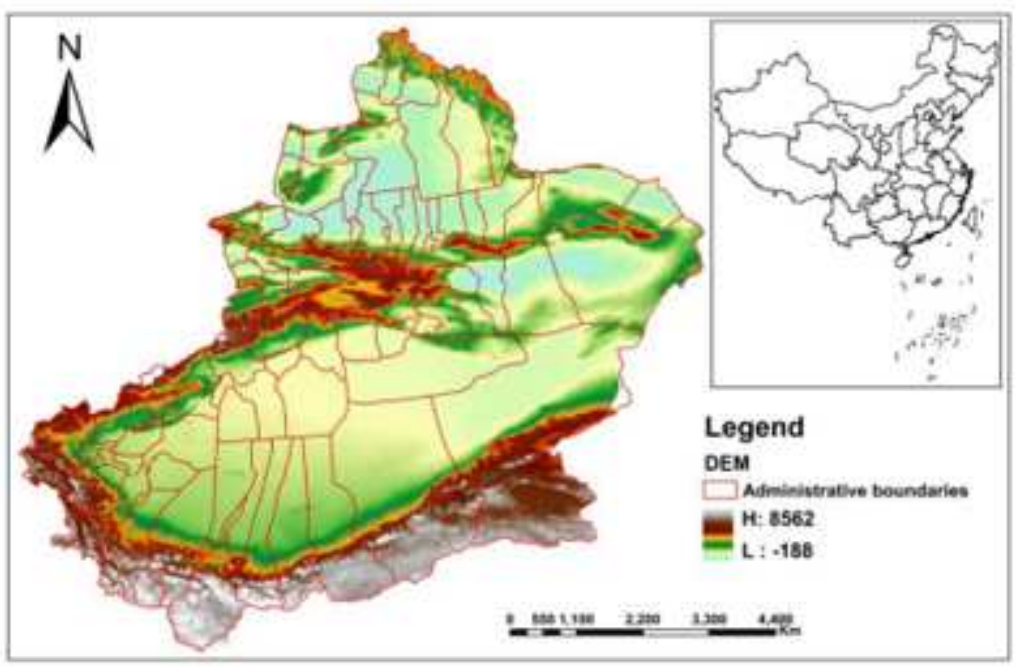

\section{Figure 1}

Geographical location of the study area in China Note: The designations employed and the presentation of the material on this map do not imply the expression of any opinion whatsoever on the part of Research Square concerning the legal status of any country, territory, city or area or of its authorities, or concerning the delimitation of its frontiers or boundaries. This map has been provided by the authors. 

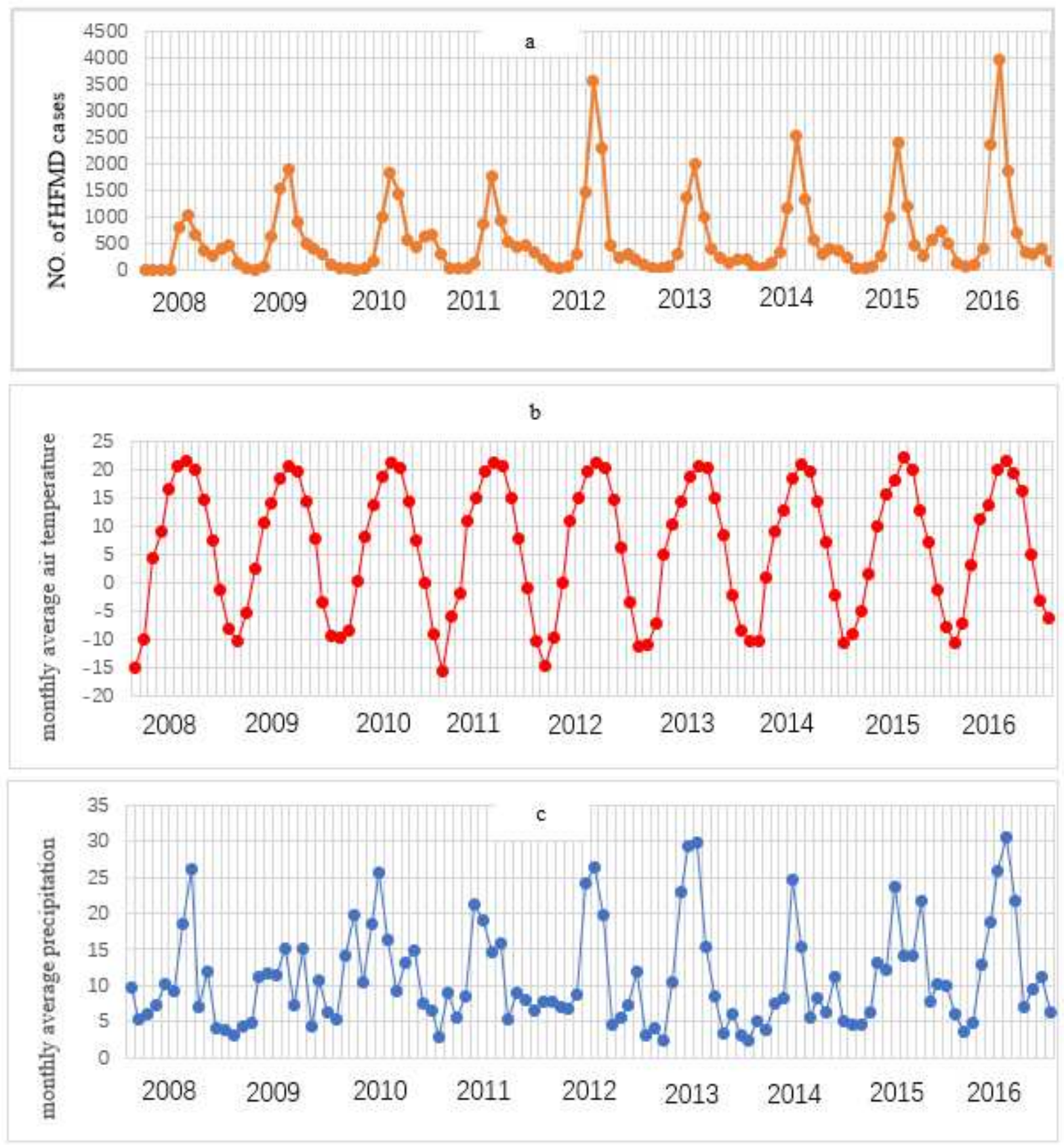

Figure 2

Time series of monthly data of HFMD cases (a), temperature (b) and precipitation (c) in Xinjiang, China from 2008 to 2016 (unit: Temperature $\left({ }^{\circ} \mathrm{C}\right)$, precipitation $(\mathrm{mm})$ ) 

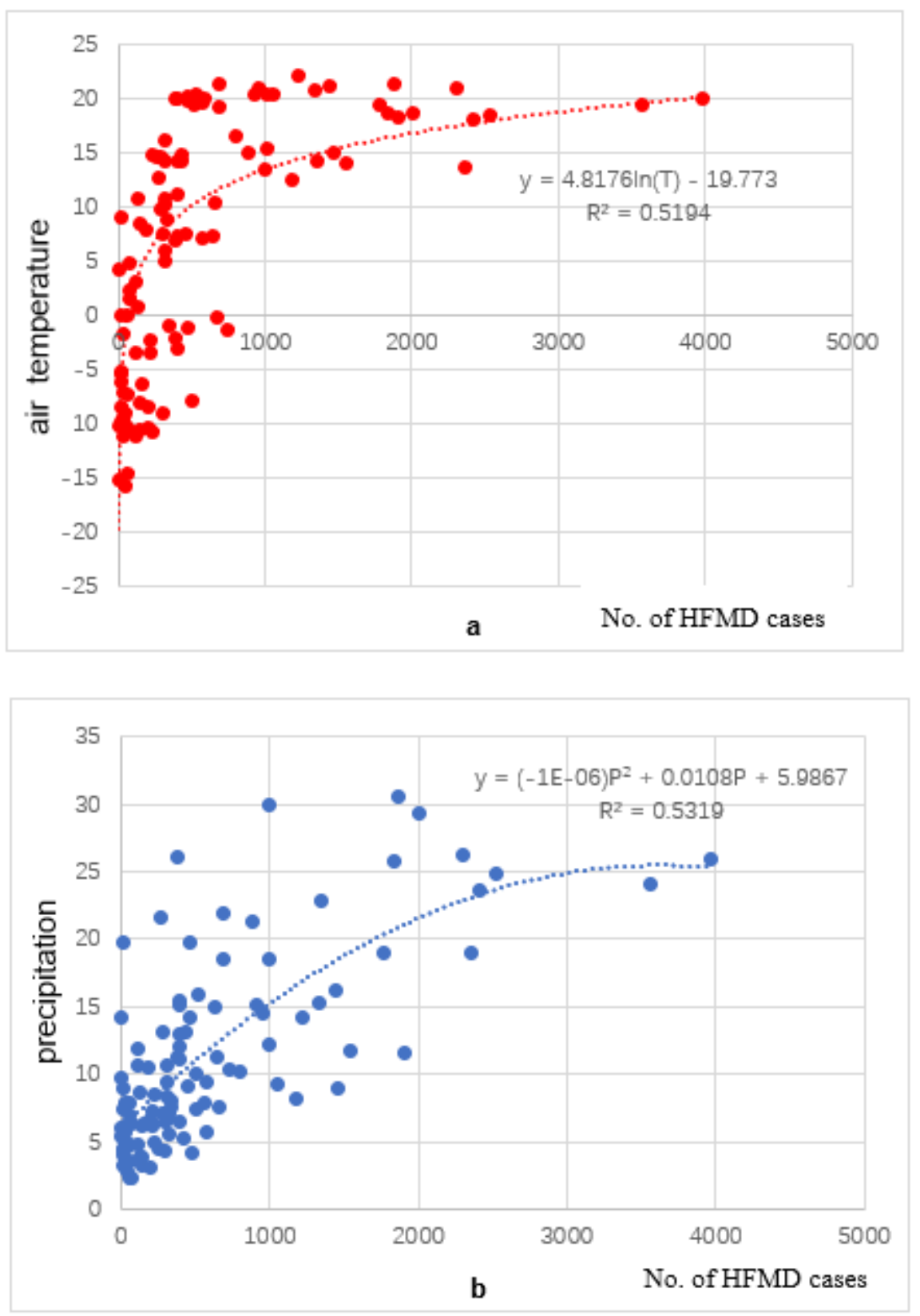

\section{Figure 3}

Fitting curve of meteorological factors (mean monthly temperature (a) and mean monthly precipitation (b)) and HFMD cases (unit: temperature $\left({ }^{\circ} \mathrm{C}\right)$, precipitation $(\mathrm{mm})$ ). 

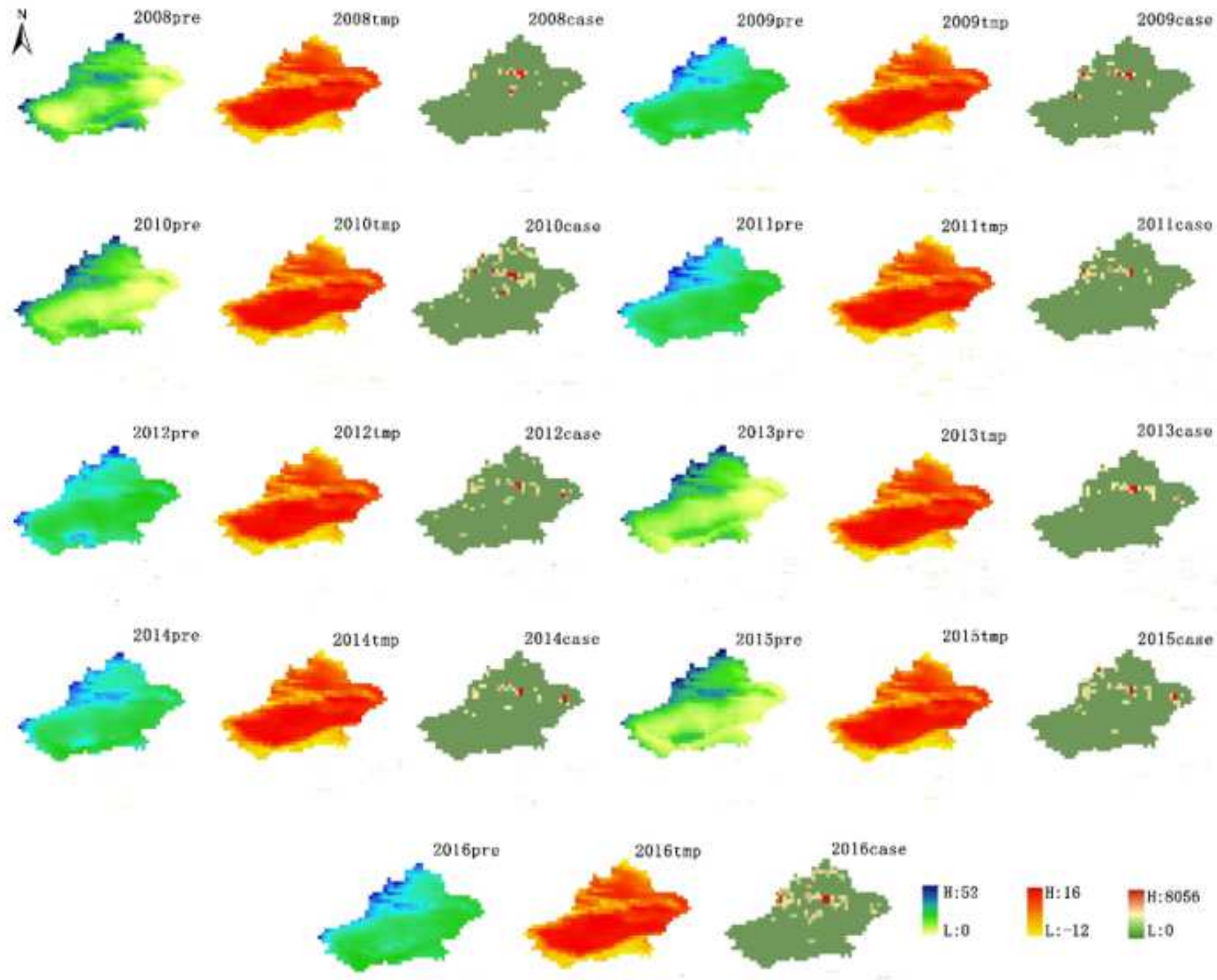

H:8056

$0 \quad 700 \quad 1,400$

Figure 4

Raster data of mean annual temperature, precipitation and HFMD cases in the study area from 2008 to 2016. 

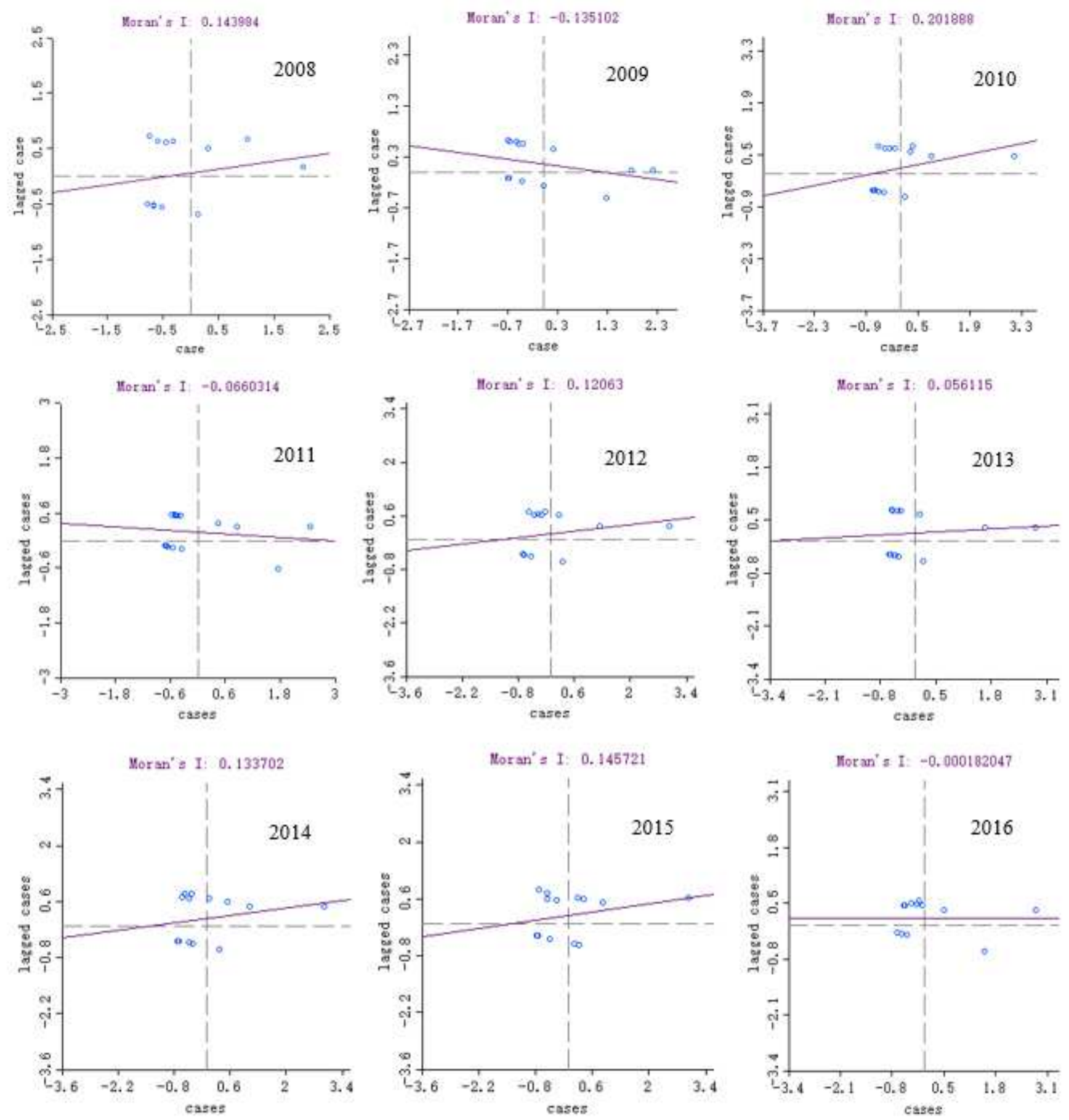

\section{Figure 5}

Moran scatter diagram of Xinjiang HFMD from 2008 to 2016. 